\title{
DISPERSION ANALYSIS IN RADAR TOMOGRAPHY
}

\author{
S. TILLARD*, S. AHR*, F. HOLLENDER*, F. JULLIEN** \\ * CEA/DCC \\ Département d'Etudes Entreposage et Stockage des Déchets \\ C.E. CADARACHE - 13108 St Paul lez Durance cedex - France \\ ** ANDRA \\ Parc de la Croix Blanche \\ 92298 Châtenay Malabry - France
}

\section{Introduction}

Ground Penetrating Radar and Borehole Radar are commonly used for various applications in civil engineering, archeology, aquifer and soil studies... These prospection methods can also be used to study geological formations for underground waste disposal, for which a set of specifications has been defined by ANDRA. In electrically resistive host rock, for example granite, they allow the localization of discontinuities such as fractures or altered zones.

Radar data collected in reflection or transmission mode can be interpreted. The rock volume which is expected to be investigated by radar through non-destructive testing is $100 \mathrm{~m}$ large around a single hole or 150 to $200 \mathrm{~m}$ large, between two boreholes.

Our study concerns tomography: tomographic imaging is achieved by transmitting signals along as many different paths as possible through the area to be explored. The area, cut into cells, is limited by two coplanar boreholes. The transmitting antenna is placed in one borehole, the receiving antenna in the second borehole. From each ray (or recorded propagated signal), one parameter is extracted. Tomography consists in solving an inverse problem to obtain the value of the parameter in each cell. At present, the parameters commonly extracted from radar signals are pick-to-pick amplitudes and first arrival times. The resulting maps are attenuation tomography and velocity tomography. These analyses do not take into account dispersion of radar signals. Moreover, uncertainties inherent in instrumental drifts (power supply, time basis ...) may lead to inexact interpretation.

\section{Principle of dispersion tomography and first tests}

In the Fourier domain, the general form of a radar wave propagating in one $\mathrm{D}$ is :

$$
E(x, \omega)=E_{0}(\omega) e^{-i x x}
$$


with

$$
k=\omega \sqrt{\mu \varepsilon_{e}}
$$

in which $E(x, \omega)$ is the electric field spectrum at any $x$ coordinate, $E_{0}(\omega)$ the electric field spectrum at $x=0, \omega$ the angular frequency, $k$ the wave number, $\mu$ the magnetic permeability ( $\mu=\mu_{0}$, the free space permeability), and $\varepsilon_{e}$ the effective dielectric permittivity.

$\varepsilon_{e}$ is a complex quantity, frequency dependent, including conduction losses in its imaginary component. This frequency dependence leads to radar dispersion. We have shown that $\varepsilon_{e}(\omega)$ can be described by a power law, established from Jonscher results (1977) and validated on laboratory measurements. The power law is:

$$
\varepsilon_{e}(\omega)=\varepsilon_{0} \chi_{r}\left(\frac{\omega}{\omega_{r}}\right)^{n-1}\left[1-i \cot \left(\frac{n \pi}{2}\right)\right]+\varepsilon_{\infty},
$$

in which $\varepsilon_{\infty}$ is the high frequency limit value of the dielectric permittivity, $\chi_{r}$ the real part of the electric susceptibility at the reference frequency $\omega_{r}$, and $0<n<1$.

Thus, three real and constant parameters can describe the effective dielectric permittivity variations in the radar frequency range: $\varepsilon_{\infty}, \chi_{r}$ and $n$. This triplet can be changed by $\left(\varepsilon_{\infty}, \chi_{r}, Q_{r}\right)$ with $Q_{r}$ the quality factor at the référence frequency $\omega_{r}$.

Dispersion tomographies consist in extracting the Jonscher parameters from radar signals. These parameters being characteristic of the investigated medium geology, we should be able to provide maps of $\varepsilon_{e}$ variations for the prospected area. Then, these variations will have to be linked to geological discontinuities (see below).

To extract the Jonscher parameters (or to achieve a wavelet inversion), we follow the forward modeling principle: we have to compare an observed wavelet with synthetic wavelets generated by different Jonscher triplets. This is illustrated in Figure 1 in which a visual fitting allowed us to know the Jonscher parameters relative to three geological formations. In order to generalize this kind of comparison to many traces as it should be achieved in tomography operation, two problems have to be solved.

1- We have to minimize the misfit between observed and modeled wavelets by calculating error functions. Our first results were obtained with the Normalized Crosscorrelation Energy Function proposed by Sen and Stoffa (1995) and applied on centered, normalized wavelets. In this way, uncertainties due to radar equipment limits mentionned in the introduction should be avoided. 
2- We have to choose a proper protocole to define the Jonscher parameter space and to explore it as quickly as possible. At present, the Grid Search technique was adopted.

Up to now, this inversion scheme has been tested on noisy synthetic data. To be efficient on field data, we have to proceed with wavelet separation techniques to correctly invidualize the wavelet to invert.

The waveform analysis described here is applied for a homogeneous medium, characterized by a single Jonscher triplet. We have shown that this analysis is still valid for a heterogeneous medium, divided into homogeneous cells. Empiric linear relations for each parameter are established :

$$
\frac{1}{L_{k}} \sum_{i=1}^{n} l_{i k} \frac{1}{Q_{r}^{i}}=\frac{1}{\tilde{Q_{r}^{k}}} \text { (4), } \frac{1}{L_{k}} \sum_{i=1}^{n} l_{i k} \sqrt{\varepsilon_{\infty}^{i}}=\sqrt{\tilde{\varepsilon}_{\infty}^{k}} \text {, (5) and } \frac{1}{L_{k}} \sum_{i=1}^{n} l_{i k} \chi_{r}^{i}=\tilde{\chi}_{r}^{k},
$$

in which $n$ is the cell number, $l_{i k}$ the distance covered by the $k$ ray in the $i$ cell, $L_{k}$ the total distance covered by the $k$ ray $\left(L_{k}=\sum_{i=1}^{n} l_{i k}\right), Q_{r}^{i}$ (respectively $\left.\varepsilon_{\infty}^{i}, \chi_{r}^{i}\right)$ the quality factor at the reference frequency $\omega_{r}$ (respectively the high frequency permittivity value and the real part of the electric susceptibility at a reference frequency $\left.\omega_{r}\right)$ of the $i$ cell $\left(1 / Q_{r}^{i}, \varepsilon_{\infty}^{i}, \chi_{r}^{i}\right.$ are the parameters we are looking for in the inversion), $\tilde{Q}_{r}^{k}$ (respectively $\widetilde{\varepsilon}_{\infty}^{k}, \tilde{\chi}_{r}^{k}$ ) the quality factor (respectively the high frequency permittivity value, and the real part of the electric susceptibility) found through the inversion of the $k$ ray.

Figure $2 \mathrm{~b}$ gives an example of a $Q$ tomography performed by using the Conjugate Gradient inversion method. Our field model is shown in Figure 2a. Next step will consist in validating this new approach of radar tomography on field data. We will use data recorded in boreholes drilled in granite in an underground laboratory, the Grimsel Test Site in the Central Swiss Alps. Finally, since the aim of such studies is the interpretation of the $\varepsilon_{\infty}, \chi_{r}$, and $Q$ maps into petrophysical information, effective dielectric permittivity laboratory measurements are carried out on rock samples collected on borehole cores, for which mineralogical analyses are available.

\section{References}

Jonscher A.K., 1977, The universal dielectric response, Nature, 267, 673-679.

Sen M., Stoffa P.L., 1995, Global optimization methods in geophysical inversion, Advances in exploration geophysics 4, Elsevier. 

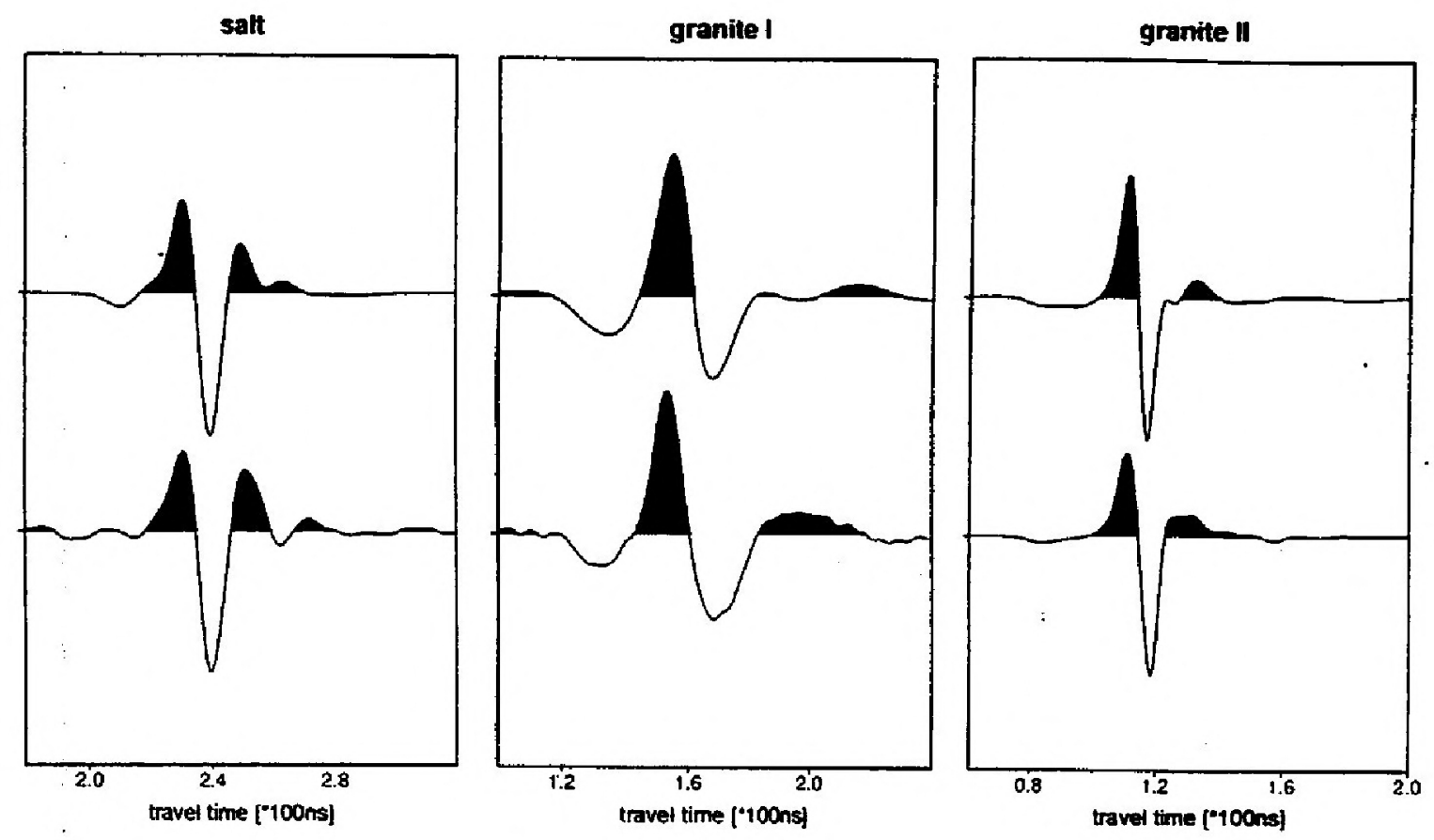

Figure 1: modeled (top) and field (bottom) wavelet recorded in transmission in salt and in two different granitic formations (granite I and granite II). The corresponding Jonscher triplets $\left(\varepsilon_{\infty}, \chi_{r}, Q_{r}\right)$ are:

$(1.3,5,30),(1.4,5,6.5),(1.4,4.8,13)$ respectively for salt, granite I; and granite II.
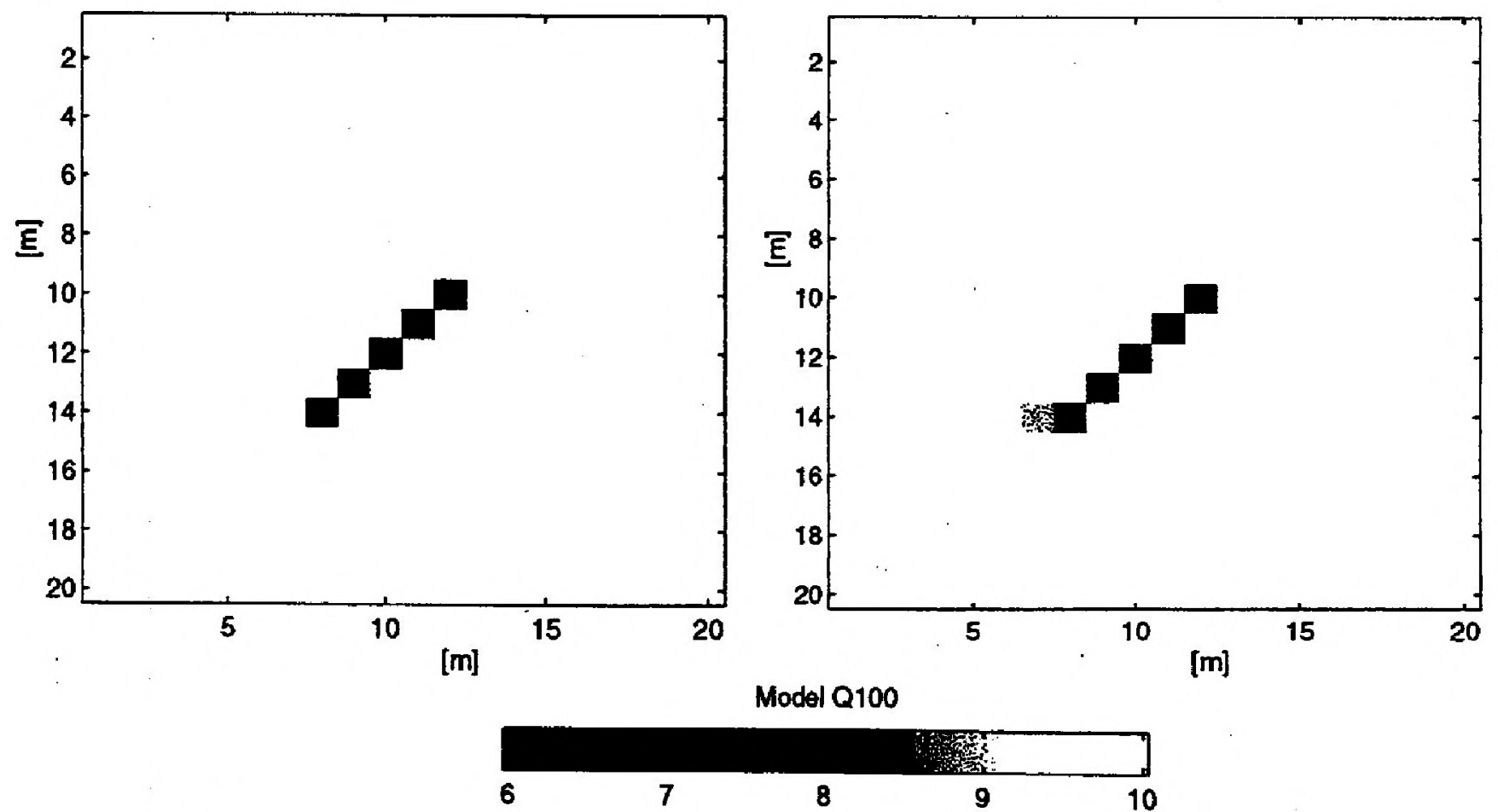

(a)

(b)

Figure 2: (a) Field model, (b) Tomography calculated by the Conjugate Gradient method 